\title{
含氟喹啉酰胺类化合物的合成及杀菌活性
}

\author{
倪 芸 ${ }^{a}$ 许天明*, $b$ 钟良坤 ${ }^{a}$ 孔晓燕 ${ }^{a}$ 史建俊 ${ }^{a}$ 刘幸海 ${ }^{a}$ \\ 孔小林 $b$ 姬文娟 ${ }^{b}$ 谭成侠 $*, a$ \\ ( ${ }^{a}$ 浙江工业大学化学工程学院 杭州 310014) \\ ( ${ }^{b}$ 中化蓝天浙江省化工研究院有限公司 杭州 310023)
}

\begin{abstract}
摘要 为寻求具有较高生物活性的农药先导化合物, 笔者采用活性亚结构拼接方法设计合成一类含氟喹啉酰胺类化合 物. 以 5-氯靛红与 2,6-二氟苯乙酮为起始原料, 经过环合、酰化等多步反应, 得到 13 个未见文献报道的含氟喹啉酰胺 类化合物. 其结构均经 ${ }^{1} \mathrm{H}$ NMR 和 HRMS 确证. 初步生物活性测定结果表明, 在 $50 \mathrm{mg} / \mathrm{L}$ 质量浓度下, 目标化合物对小 麦全蚀病(Gaeumannomyces graminis)、小麦赤霉病(Fusahum graminearum sehw)、小麦纹枯病(Rhizoctonia cerealis)、水 稻稻瘟病(Pyricularia grisea)均具有杀菌活性. 而部分化合物对小麦全蚀病(Gaeumannomyces graminis)的杀菌活性达到 $90 \%$ 以上.
\end{abstract}

关键词 含氟喹啉; 酰胺类化合物; 合成; 杀菌活性

\section{Synthesis and Fungicidal Activity of a Series of Fluorinated Quinoline Amide Compounds}

\author{
Ni, Yun ${ }^{a} \quad \mathrm{Xu}$, Tianming $^{*, b} \quad$ Zhong, Liangkun ${ }^{a}$ \\ Kong, Xiaoyan ${ }^{a}$ \\ Liu, Xinghai $^{a} \quad K$ Kong, Xiaolin ${ }^{b} \quad$ Ji, Wenjuan $^{b} \quad$ Tan, Chengxia*,a $^{*}$ \\ ( ${ }^{a}$ College of Chemical Engineering, Zhejiang University of Technology, Hangzhou 310014) \\ ( ${ }^{b}$ Sinochem Lantian Zhejiang Research Institute of Chemical Industry Co. Ltd, Hangzhou 310023)
}

Shi, Jianjun ${ }^{a}$

\begin{abstract}
To find new fungicidal lead compounds with high bioactivity, novel lead compounds were designed by active group splicing. The synthetic routes are as follows: 6-chloro-2-(2,6-difluorophenyl)quinoline-4-carbonyl chloride (7) was synthesized using 5-chlorine isatin (4) and 2,6-difluoro acetophenone (5) as raw materials by cyclization, acyl chlorination and other step reactions. Then 13 novel title compounds were synthesized by acylation of 6-chloro-2-(2,6-difluorophenyl)-quinoline-4-carbonyl chloride (7) with substituted anilines (8). The structures of the compounds were confirmed by HRMS and ${ }^{1} \mathrm{H}$ NMR. The preliminary fungicidal activity showed that all the compounds exhibit fungicidal activity against Gaeumannomyces graminis and Fusahum graminearum sehw, Rhizoctonia cerealis and Pyricularia grisea at concentration of $50 \mathrm{mg} / \mathrm{L}$, part of compounds against Gaeumannomyces graminis were more than $90 \%$ at concentration of $50 \mathrm{mg} / \mathrm{L}$.
\end{abstract}

Keywords fluorinated quinoline; amide compounds; synthesis; fungicidal activity

从 20 世纪 80 年代开始, 开发了许多高效低毒环保 的新杂环农药, 而稠杂环化合物作为杂环化合物的重要 分支已经引起人们的关注 ${ }^{[1,2]}$. 尤其是含氟稠杂环类化 合物兼具多个杂环的生物活性 ${ }^{[3]}$, 已经成为研究的热点 之一. 喹啉衍生物是一类重要的含氮稠杂环化合物, 其 在医药和农药方面都有广泛的应用, 并显示出良好的发 展前景 ${ }^{[4 \sim 6]}$. 例如, 苯氧喹啉(Quinoxyfen, 1) 就是一个高
效防治白粉病药剂 ${ }^{[7]}$. 另外由日本 Hokko 化学开发的化 合物 $\mathbf{2}^{[8]}$, 道尔化学开发的化合物 3 具有很好的杀菌活 性 ${ }^{[9]}$, 其结构式如图 1 所示. 酰胺类化合物同样表现出 优异的生物活性 ${ }^{[10]}$, 有的已经开发成为商品化的农药, 如除草剂甲草胺、杀菌剂啶酰菌胺、杀螨剂吡螨胺等.

本文为了寻求具有高活性的农药先导化合物, 采用 活性亚结构拼接方法, 将含氟稠杂环喹啉环与酰胺结构

\footnotetext{
* E-mail: tanchengxia@zjut.edu.cn

Received April 18, 2015; revised June 3, 2015; published online June 9, 2015.

Project supported by the National Key Technologies R\&D Program (No. 2011BAE06B02-04).

“十二五”国家科技支撑计划(No. 2011BAE06B02-04)资助项目.
} 
<smiles>Fc1ccc(Oc2ccnc3cc(Cl)cc(Cl)c23)cc1</smiles><smiles>O=C(Nc1cnc2ccccc2c1)C1(C2(O)CCCCCC2)OCCS1</smiles><smiles>CC(C)(C)c1ccc(CC(=O)Nc2ccnc3c(F)cccc23)cc1</smiles>

图 1 喹啉类化合物

Figure 1 Quinoline compounds

相结合, 设计并合成出一系列具有新颖结构的喹啉酰胺 类化合物，并通过核磁共振、高分辨质谱进行结构表征， 生物活性测试表明部分化合物具有较好的杀菌活性. 目 标化合物合成路线如 Scheme 1 所示.<smiles>O=C1Nc2ccc(Cl)cc2C1=O</smiles>

4<smiles>O=C(O)c1cc(-c2c(F)cccc2F)nc2ccc(Cl)cc12</smiles>

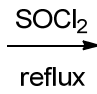<smiles>O=C(Cl)c1cc(-c2c(F)cccc2F)nc2ccc(Cl)cc12</smiles>

$+\mathrm{RNH}_{2}$

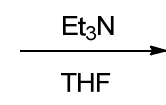

7

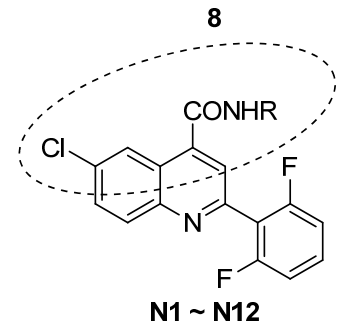

$\mathrm{R}=2-\mathrm{Me}-4-\mathrm{CF}\left(\mathrm{CF}_{3}\right)_{2} \mathrm{C}_{6} \mathrm{H}_{3}, 2,5-\mathrm{Me}_{2} \mathrm{C}_{6} \mathrm{H}_{3}, 2-\mathrm{F}-4-\mathrm{t}-\mathrm{Bu}-\mathrm{C}_{6} \mathrm{H}_{3}$, 3- $\mathrm{MeOC}_{6} \mathrm{H}_{4}, 4-\mathrm{BrC}_{6} \mathrm{H}_{4}, 4-\mathrm{MeC}_{6} \mathrm{H}_{4}, 4-\mathrm{EtC}_{6} \mathrm{H}_{4}, 2-\mathrm{CNC}_{6} \mathrm{H}_{4}$, 3- $\mathrm{CNC}_{6} \mathrm{H}_{4}, \mathrm{CH}_{2} \mathrm{C}_{6} \mathrm{H}_{4}, 2-\mathrm{FC}_{6} \mathrm{H}_{4}, 4-\mathrm{FC}_{6} \mathrm{H}_{4}, 2-\mathrm{ClC}_{6} \mathrm{H}_{4}$

图式 1 喹啉酰胺类化合物合成路线

Scheme 1 Synthetic route of quinoline amide compounds

\section{1 结果与讨论}

\section{1 合成及波谱数据}

中间体 6 的合成参考文献[11]. 以 5-氯靛红与取代 苯乙酮反应, 形成喹啉环. 如 Scheme 2 所示为喹啉环形 成的机理, 5-氯靛红在碱性条件下发生水解, 生成酮酸 中间体，在与苯乙酮发生缩合反应形成亚胺结构中间 体，经过互变异构，生成烯胺，最后，烯胺分子内关环 脱水得到最终产物. 对文献中使用的酸性条件进行改 进，本文使用碱性条件反应，纯度较高，产率达到 $92.3 \%$. 而且实验过程中使用质子性溶剂水作为反应介 质，无有机溶剂，清洁环保，后处理简单.<smiles>O=C1Nc2ccc(Cl)cc2C1=O</smiles><smiles>CC(=O)c1c(F)cccc1F</smiles><smiles>C/C(=N\c1ccc(Cl)cc1C(=O)C(=O)O)c1c(F)cccc1F</smiles><smiles>CCCCC</smiles><smiles>O=C(O)C(=O)Nc1ccc(Cl)cc1C(=O)Nc1cccc(F)c1</smiles><smiles>O=C(O)c1cc(-c2c(F)cccc2F)nc2ccc(Cl)cc12</smiles>

图式 2 喹啉环成环机理

Scheme 2 Mechanism of cyclization of cyslization

中间体 7 的合成参考文献[12]. 该反应为酰化反应, 反应需要加热回流, 以便反应完全, 提高产率. 反应结 束后必须蒸馏出剩余的二氯亚砜, 避免二氯亚砜的残留 对下一步的反应产生影响，降低产率.

目标化合物 N1 N N13 的合成参考文献[13]. 部分反 应物因含有 $\mathrm{CN}$ 等强吸电子基团使苯环针化, 反应收率 下降, 又因为 2 位引入 $\mathrm{CN}$ 可能存在位阻效应, 产率较 其他位置会更低. 并且反应时间不宜过长, 过长会产生 副产物，也会降低反应产率.

在 ${ }^{1} \mathrm{H}$ NMR 谱中, 目标化合物喹啉环上的四个 $\mathrm{H}$ 的 $\delta$ 值主要在 $7.77 \sim 8.40$ 之间，其中 7 位上 $\delta 7.77$ 左右的 $\mathrm{H}$ 峰型是 $\mathrm{dd}$ 峰, 此位置上的 $\mathrm{H}$ 可能受喹啉环 8 位和 5 位 上的 $\mathrm{H}$ 偶合的影响. 喹啉环 5 位上的 $\delta$ 值主要在 8.40 左右, 峰型为 $\mathrm{d}$ 峰, 此位置上的 $\mathrm{H}$ 可能受喹啉环 7 位上 的 $\mathrm{H}$ 偶合的影响. 喹啉环 8 位上的 $\delta$ 值主要在 8.20 左右, 
峰型为 $\mathrm{d}$ 峰, 此位置上的 $\mathrm{H}$ 可能受喹啉环 7 位上的 $\mathrm{H}$ 偶 合的影响. 与 $\mathrm{NH}$ 直接相连的苯环上的 $\mathrm{H}$ 的 $\delta$ 值变化较 大, 主要在 $\delta 7.10$ 和 7.60 左右, 且为多重峰. 酰胺上的 $\mathrm{H}$ 主要出现在 $\delta 8.00$ 左右, 峰型为单峰. 此类化合物特 殊的立体结构以及构效关系, 有待进一步研究.

\section{2 杀菌活性}

参照《国家南方农药创制中心生测标准程序》 ${ }^{[14]}$, 准确称取(精确至 $0.0001 \mathrm{~g}$ ) 一定质量目标化合物, 加入 含有 $0.1 \%$ 吐温- 80 的 $N, N$-二甲基甲酰胺(DMF), 配制成 化合物质量分数为 $5 \%$ 的母液. 称取一定质量的制剂和 对照药剂, 分别用蒸馏水稀释成 $50 \mathrm{mg} / \mathrm{L}$ 质量浓度的药 液. 采用菌丝生长法, 在离体条件下测定了目标化合物 在 $50 \mathrm{mg} / \mathrm{L}$ 下对病原菌的杀菌活性. 以三唑酮、苯醚菌 酯为对照药剂(用蒸馏水稀释成有效成分质量浓度为 50 $\mathrm{mg} / \mathrm{L})$, 同时设置清水对照.

供试靶标选取的是小麦全蚀病(Gaeumannomyces graminis)、小麦赤霉病(FusaHum graminearum Sehw)、 小麦纹枯病 (Rhizoctonia cerealis)、水稻稻瘟病 (Pyricularia grisea). 这四种靶标是南方田间发病较重 的, 有代表性的病原菌. 而且这四种靶标是国家南方农 药研发中心浙江基地生测测试中心使用较多的病原菌.

本文采用离体活性测试法. 吸取 $4 \mathrm{~mL}$ 由 $5 \%$ 的制剂 配制成的浓度为 $500 \mathrm{mg} / \mathrm{L}$ 的溶液到灭菌的三角烧瓶中, 加入 $36 \mathrm{~mL} 50{ }^{\circ} \mathrm{C}$ 的马铃薯葡萄糖琼脂(PDA)培养基, 将二者混合均匀后倒入至 4 个直径为 $9 \mathrm{~cm}$ 的培养血中, 配制成终浓度均为 $50 \mathrm{mg} / \mathrm{L}$ 的含毒 PDA 培养基. 用直径 $5 \mathrm{~mm}$ 的打孔器把培养好的菌落边缘打成菌块, 然后将 菌块接种至预先配制好的含毒 PDA 培养基中央, 置于 $25{ }^{\circ} \mathrm{C}$ 的培养箱内培养. 观察菌落生长状况, 采用十字 交叉法量取各处菌落的直径, 计算出正抑制百分率, 测 试结果见表 1 .

从表 1 可以看出，化合物 N5、N6、N8、N12 在 50 $\mathrm{mg} / \mathrm{L}$ 的剂量下对小麦全蚀病的抑制率分别为 $94.29 \%$ 、 $92.43 \% 、 98.43 \% 、 96.14 \%, \mathbf{N 8}$ 比 N5、N6、N12 的活性 更高, 说明 $\mathrm{R}$ 为含强吸电子基团或含卤素原子的苯环 时, 其杀菌活性明显优于其它目标化合物, 而且酰胺结 构、氟、喹啉等活性基团以及吸电子结构集于同一分子, 可获得更高活性的化合物.

\section{2 结论}

本文以 5 -氯靛红(4)为起始原料, 与 2,6 -二氟苯乙酩 (5)反应, 得到 6-氯-2-(2,6-二氟苯基)喹啉-4-甲酸(6), 再 经过酰化后与不同取代的苯胺反应, 制得一系列未见文 献报道的目标化合物: 含氟喹啉酰胺类化合物. 并对该 系列化合物通过 ${ }^{1} \mathrm{H}$ NMR 和 HRMS 进行了结构表征. 杀
表 1 目标化合物在 $50 \mathrm{~mL} / \mathrm{L}$ 下的杀菌活性(抑制率/\%)

Table 1 Fungicidal activity of $\mathrm{N}$ compounds at the concentration of $50 \mathrm{mg} / \mathrm{L}$ (Inhibition rate)

\begin{tabular}{ccccc}
\hline Compd. & G. graminis & F. sehw & R. cerealis & P. grisea \\
\hline N1 & 61.31 & 21.12 & 21.45 & 46.17 \\
$\mathbf{N 2}$ & 35.45 & 15.23 & 19.47 & 20.78 \\
$\mathbf{N 3}$ & 70.46 & 12.56 & 29.14 & 54.87 \\
$\mathbf{N 4}$ & 26.42 & 12.89 & 20.54 & 36.10 \\
$\mathbf{N 5}$ & 94.29 & 18.10 & 32.35 & 70.92 \\
$\mathbf{N 6}$ & 92.43 & 25.88 & 34.47 & 76.79 \\
$\mathbf{N} 7$ & 84.57 & 16.69 & 32.19 & 61.49 \\
$\mathbf{N 8}$ & 98.43 & 76.73 & 31.04 & 53.54 \\
$\mathbf{N 9}$ & 56.86 & 20.05 & 28.16 & 27.04 \\
$\mathbf{N 1 0}$ & 69.14 & 35.82 & 31.04 & 60.79 \\
$\mathbf{N 1 1}$ & 12.86 & 25.88 & 21.90 & 59.67 \\
$\mathbf{N 1 2}$ & 96.14 & 59.36 & 22.16 & 79.42 \\
N13 & 20.86 & 15.54 & 33.11 & 21.04 \\
& & & & \\
三唑酮 & 100 & 100 & 100 & \\
(triadimefon) & & & & \\
苯醚菌酯 & & & & \\
(ZJ0712) & & & & \\
空白对照 & 0 & 0 & & \\
\hline
\end{tabular}

菌活性测试表明，在 $50 \mathrm{mg} / \mathrm{L}$ 浓度下，一些目标化合物 对小麦全蚀病有优异的抑制活性，部分小麦赤霉病、水 稻稻瘟表现出中等抑制活性.

\section{3 实验部分}

\section{1 仪器与试剂}

WRS-1A 型数字熔点仪(温度计未校正); Varian-400 型核磁共振仪 (以 DMSO 作溶剂, 以 TMS 为内标); LCQAdvantage 质谱仪(ESI-MS); 5-氯-1 $H$-吲哚-2,3-二酮(试 剂级，上海迈瑞尔化学技术有限公司); 2,6-二氟苯乙酮 (试剂级, 上海迈瑞尔化学技术有限公司). 其余试剂均 为化学纯或试剂纯, 未经处理, 直接使用.

\section{2 化合物的合成}

\subsubsection{6-氯-2-(2,6-二氟苯基)喹啉-4-甲酸(6)的合成}

参考文献[11]方法. 向 $500 \mathrm{~mL}$ 三口烧瓶中加入 $47.04 \mathrm{~g}(0.84 \mathrm{~mol})$ 氢氧化钾, 加入 $150 \mathrm{~mL}$ 去离子水, 室 温下搅拌. 向反应瓶中再加入 $25.41 \mathrm{~g}(0.14 \mathrm{~mol}) 5$-氯 - $1 H$-吲哚-2,3-二酮(4), 补加 $30 \mathrm{~mL}$ 去离子水后, 接着 向反应瓶中加入 $21.84 \mathrm{~g}(0.14 \mathrm{~mol}) 2,6$-二氟苯乙酩(5), 加热回流 $3 \mathrm{~h}$, 薄层层析(TCL)检测无原料点. 将反应液 倒入 $800 \mathrm{~mL}$ 水中, 搅拌, 加入稀盐酸, 调节 $\mathrm{pH}$ 值至 5 , 过滤, 滤饼用去离子水洗涤 2 次, 烘干, 得到 $41.3 \mathrm{~g}$ 黄色 固体 6, 产率 92.3\%. m.p. $251 \sim 256{ }^{\circ} \mathrm{C} ;{ }^{1} \mathrm{H}$ NMR (400 MHz, DMSO- $\left.d_{6}\right) \delta: 7.33$ (t, $\left.J=8.0 \mathrm{~Hz}, 2 \mathrm{H}, \mathrm{ArH}\right), 7.61 \sim$ 
7.67 (m, 1H, ArH), 7.94 (d, $J=8.0 \mathrm{~Hz}, 1 \mathrm{H}$, quinoline-H), $7.74(\mathrm{~d}, \quad J=8.0 \mathrm{~Hz}, 1 \mathrm{H}$, quinoline- $\mathrm{H}), 8.19 \sim 8.21(\mathrm{~m}$, $2 \mathrm{H}$, quinoline- $\mathrm{H}), 8.88$ (s, $1 \mathrm{H}$, quinoline- $\mathrm{H})$.

\subsubsection{6-氯-2-(2,6-二氟苯基)喹啉-4-酰氯(7)的合成}

参考文献[12]方法进行. 将 $35.16 \mathrm{~g}(0.11 \mathrm{~mol})$ 化合 物 6 加入 $500 \mathrm{~mL}$ 单口烧瓶中, 再加入 $200 \mathrm{~mL}$ 二氯亚砜, 加热回流 $5 \mathrm{~h}, \mathrm{TLC}$ 检测反应进度, 反应结束后, 常压蒸 馏出多余二氯亚砜, 得到黄色固体 7. m.p. $124 \sim 129{ }^{\circ} \mathrm{C}$. 不经提纯直接用于下一步的反应.

\subsection{3 目标化合物 N1 N13 的合成通法}

参考文献[13]的方法, 向 $50 \mathrm{~mL}$ 单口烧瓶中加入 $0.42 \mathrm{~g}(0.01 \mathrm{~mol})$ 化合物 7, 加入 $15 \mathrm{~mL}$ 四氢呋喃溶解, 再加入 $0.01 \mathrm{~mol}$ 的取代芳基胺和 $0.02 \mathrm{~mol}$ 的三乙胺. 室 温搅拌反应 $5 \mathrm{~h}$. TLC 检测反应进度, 反应结束后, 过滤, 脱溶得固体, 无水乙醇重结晶即得目标化合物 $\mathbf{N} 1 \sim$ N13.

6-氯-2-(2,6-二氟苯基)- $N$-[2-甲基-4-(全氟丙烷-2-基) 苯基]喹啉-4-甲酰胺(N1): 淡黄色晶体, 收率 68\%. m.p. $201 \sim 205{ }^{\circ} \mathrm{C} ;{ }^{1} \mathrm{H}$ NMR $\left(\mathrm{CDCl}_{3}, 400 \mathrm{MHz}\right) \delta: 2.40$ (s, 3H, $\mathrm{ArCH}_{3}$ ), 7.09 (t, $\left.J=8.0 \mathrm{~Hz}, 2 \mathrm{H}, \mathrm{ArH}\right), 7.43 \sim 7.59(\mathrm{~m}, 3 \mathrm{H}$, ArH), 7.75 (s, 1H, quinoline-H), $7.78(\mathrm{dd}, J=8.0,1.2 \mathrm{~Hz}$, $1 \mathrm{H}$, quinoline-H), 8.19 (d, $J=8.0 \mathrm{~Hz}, 1 \mathrm{H}$, quinoline-H), $8.36(\mathrm{~d}, J=4.0 \mathrm{~Hz}, 1 \mathrm{H}, \operatorname{ArH}), 8.40(\mathrm{~d}, J=1.2 \mathrm{~Hz}, 1 \mathrm{H}$, quinoline-H), $7.81(\mathrm{~s}, 1 \mathrm{H}, \mathrm{CONH})$; HRMS calcd for $\mathrm{C}_{26} \mathrm{H}_{14} \mathrm{ClF}_{9} \mathrm{~N}_{2} \mathrm{O}$ 577.0724, found 577.0765.

6-氯-2-(2,6-二氟苯基)- $N$-(2,5-二甲苯基)喹啉-4-甲 酰胺(N2): 淡黄色晶体, 收率 $80 \%$. m.p. $236 \sim 240{ }^{\circ} \mathrm{C}$; ${ }^{1} \mathrm{H} \mathrm{NMR}\left(\mathrm{CDCl}_{3}, 400 \mathrm{MHz}\right) \delta$ : 2.30 (s, 3H, $\left.\mathrm{ArCH}_{3}\right), 2.40$ (s, $3 \mathrm{H}, \mathrm{ArCH}_{3}$ ), $7.00 \sim 7.16(\mathrm{~m}, 4 \mathrm{H}, \mathrm{ArH}), 7.42 \sim 7.47$ (m, 1H, ArH), 7.58 (s, 1H, ArH), 7.77 (dd, $J=8.0,1.2 \mathrm{~Hz}, 1 \mathrm{H}$, quinoline-H), $7.81(\mathrm{~s}, 1 \mathrm{H}$, quinoline-H), 8.18 (d, $J=8.0$ $\mathrm{Hz}, 1 \mathrm{H}$, quinoline- $\mathrm{H}), 8.45(\mathrm{~d}, J=1.2 \mathrm{~Hz}, 1 \mathrm{H}$, quinoline-H), 7.88 (s, 1H, CONH); HRMS calcd for $\mathrm{C}_{24} \mathrm{H}_{17} \mathrm{ClF}_{2} \mathrm{~N}_{2} \mathrm{O} 423.1070$, found 423.1112 .

6-氯-2-(2,6-二氟苯基)- $N$-(2-氟-4-叔丁基苯基)喹啉4-甲酰胺 (N3): 淡黄色晶体, 收率 79\%. m.p. 166 $170{ }^{\circ} \mathrm{C} ;{ }^{1} \mathrm{H}$ NMR $\left(\mathrm{CDCl}_{3}, 400 \mathrm{MHz}\right) \delta: 1.34$ (s, 9H, $\left.\mathrm{CH}_{3}\right)$, 7.06 7.20 (m, 3H, ArH), 7.26 (s, $1 \mathrm{H}, \mathrm{ArH}), 7.43 \sim 7.45$ (m, 1H, ArH), 7.76 (dd, $J=8.0,1.6 \mathrm{~Hz}, 1 \mathrm{H}$, quinoline-H), 7.79 (s, 1H, quinoline-H), $7.96(\mathrm{~s}, 1 \mathrm{H}, \mathrm{CONH}), 8.18$ (d, $1 \mathrm{H}, J=8.0 \mathrm{~Hz}$, quinoline-H), $8.34 \sim 8.37(\mathrm{~m}, 1 \mathrm{H}, \mathrm{ArH})$, $8.40(\mathrm{~d}, J=1.6 \mathrm{~Hz}, 1 \mathrm{H}$, quinoline-H); HRMS calcd for $\mathrm{C}_{26} \mathrm{H}_{20} \mathrm{ClF}_{3} \mathrm{~N}_{2} \mathrm{O} 469.1289$, found 469.1318 .

6-氯-2-(2,6-二氟苯基)- $N$-(3-甲氧苯基)喹啉-4-甲酰 胺(N4)：淡黄色晶体，收率 $68 \%$. m.p. $219 \sim 224{ }^{\circ} \mathrm{C} ;{ }^{1} \mathrm{H}$
NMR $\left(\mathrm{CDCl}_{3}, 400 \mathrm{MHz}\right) \delta: 3.87\left(\mathrm{~s}, 3 \mathrm{H}, \mathrm{OCH}_{3}\right), 6.79(\mathrm{dd}$, $J=4.0,1.6 \mathrm{~Hz}, 1 \mathrm{H}, \mathrm{ArH}), 7.07$ (t, $J=8.0 \mathrm{~Hz}, 2 \mathrm{H}, \mathrm{ArH})$, $7.16(\mathrm{~d}, J=4.0 \mathrm{~Hz}, 1 \mathrm{H}, \operatorname{ArH}), 7.31(\mathrm{t}, J=12.0 \mathrm{~Hz}, 1 \mathrm{H}$, ArH), $7.41 \sim 7.46(\mathrm{~m}, 2 \mathrm{H}, \operatorname{ArH}), 7.76 \sim 7.77(\mathrm{~m}, 2 \mathrm{H}$, quinoline-H), 7.88 (s, 1H, CONH), 8.17 (d, $J=8.0 \mathrm{~Hz}, 1 \mathrm{H}$, quinoline-H), $8.37(\mathrm{~d}, J=1.6 \mathrm{~Hz}, 1 \mathrm{H}$, quinoline-H); HRMS calcd for $\mathrm{C}_{23} \mathrm{H}_{15} \mathrm{ClF}_{2} \mathrm{~N}_{2} \mathrm{O}_{2}$ 425.0863, found 425.0895 .

6-氯-2-(2,6-二氟苯基)- $N$-(4-溴苯基)喹啉-4-甲酰胺 (N5): 淡黄色晶体, 收率 62\%. m.p. $207 \sim 212{ }^{\circ} \mathrm{C} ;{ }^{1} \mathrm{H}$ NMR $\left(\mathrm{CDCl}_{3}, 400 \mathrm{MHz}\right) \delta: 7.00 \sim 7.04(\mathrm{~m}, 2 \mathrm{H}, \mathrm{ArH})$, $7.39 \sim 7.44(\mathrm{~m}, 1 \mathrm{H}, \mathrm{ArH}), 7.52(\mathrm{~d}, J=4.0 \mathrm{~Hz}, 2 \mathrm{H}, \mathrm{ArH})$, 7.60 (d, $J=4.0 \mathrm{~Hz}, 2 \mathrm{H}, \mathrm{ArH}), 7.70$ (s, $1 \mathrm{H}$, quinoline-H), 7.74 (dd, $J=8.0,1.2 \mathrm{~Hz}, 1 \mathrm{H}$, quinoline-H), 8.13 (d, $J=8.0$ $\mathrm{Hz}, 1 \mathrm{H}$, quinoline-H), 8.15 (s, $1 \mathrm{H}, \mathrm{CONH}), 8.30$ (d, $J=1.2$ $\mathrm{Hz}, 1 \mathrm{H}$, quinoline- $\mathrm{H}$ ); HRMS calcd for $\mathrm{C}_{22} \mathrm{H}_{12} \mathrm{ClF}_{2} \mathrm{~N}_{2} \mathrm{OBr}$ 473.6099, found 474.9872 .

6-氯-2-(2,6-二氟苯基)- $N$-(4-甲苯基)喹啉-4-甲酰胺 (N6): 淡黄色晶体, 收率 55\%. m.p. 229 233 ${ }^{\circ} \mathrm{C} ;{ }^{1} \mathrm{H}$ NMR ( $\left.\mathrm{CDCl}_{3}, 400 \mathrm{MHz}\right) \delta: 2.37$ (s, 3H, $\left.\mathrm{ArCH}_{3}\right), 7.04$ (t, $J=8.0 \mathrm{~Hz}, 2 \mathrm{H}, \operatorname{ArH}), 7.21(\mathrm{~d}, J=8.0 \mathrm{~Hz}, 2 \mathrm{H}, \operatorname{ArH})$, $7.40 \sim 7.43(\mathrm{~m}, 1 \mathrm{H}, \operatorname{ArH}), 7.56(\mathrm{~d}, J=8.0 \mathrm{~Hz}, 2 \mathrm{H}$, quinoline-H), $7.73 \sim 7.75$ (m, 2H, quinoline- $\mathrm{H}$ and $\mathrm{ArH})$, 7.92 (s, 1H, CONH), 8.15 (d, $J=8.0 \mathrm{~Hz}, 1 \mathrm{H}$, quinoline-H), 8.36 (s, $1 \mathrm{H}$, quinoline- $\mathrm{H}$ ); HRMS calcd for $\mathrm{C}_{23} \mathrm{H}_{15} \mathrm{ClF}_{2} \mathrm{~N}_{2} \mathrm{O}$ 409.0914, found 409.0951.

6-氯-2-(2,6-二氟苯基)- $N$-(4-乙苯基)喹啉-4-甲酰胺 (N7): 白色晶体, 收率 78\%. m.p. $163 \sim 168{ }^{\circ} \mathrm{C} ;{ }^{1} \mathrm{H}$ NMR $\left(\mathrm{CDCl}_{3}, 400 \mathrm{MHz}\right) \delta: 1.26\left(\mathrm{t}, J=8.0 \mathrm{~Hz}, 3 \mathrm{H}, \mathrm{CH}_{2} \mathrm{CH}_{3}\right)$, 2.68 (q, $\left.J=8.0 \mathrm{~Hz}, 2 \mathrm{H}, \mathrm{CH}_{2} \mathrm{CH}_{3}\right), 7.07(\mathrm{t}, J=12.0 \mathrm{~Hz}, 2 \mathrm{H}$, $\operatorname{ArH}), 7.25$ (d, $J=8.0 \mathrm{~Hz}, 2 \mathrm{H}, \mathrm{ArH}), 7.41 \sim 7.46(\mathrm{~m}, 1 \mathrm{H}$, ArH), 7.60 (d, $J=1.6 \mathrm{~Hz}, 2 \mathrm{H}$, quinoline-H), $7.75 \sim 7.77$ (m, 2H, quinoline- $\mathrm{H}$ and $\mathrm{ArH}), 7.80(\mathrm{~s}, 1 \mathrm{H}, \mathrm{CONH}), 8.17$ (d, $J=8.0 \mathrm{~Hz}, 1 \mathrm{H}$, quinoline-H), 8.39 (d, $J=1.6 \mathrm{~Hz}, 1 \mathrm{H}$, quinoline-H); HRMS calcd for $\mathrm{C}_{24} \mathrm{H}_{17} \mathrm{ClF}_{2} \mathrm{~N}_{2} \mathrm{O} 423.1070$, found 423.1109 .

6-氯-2-(2,6-二氟苯基)- $N$-(2-氰基苯基)喹啉-4-甲酰 胺(N8): 淡黄色晶体, 收率 54\%. m.p. 236 240 ${ }^{\circ} \mathrm{C} ;{ }^{1} \mathrm{H}$ NMR (DMSO- $\left.d_{6}, 400 \mathrm{MHz}\right) \delta: 7.27(\mathrm{t}, J=12.0 \mathrm{~Hz}, 3 \mathrm{H}$, ArH), $7.61 \sim 7.73(\mathrm{~m}, 2 \mathrm{H}, \mathrm{ArH}), 7.86(\mathrm{dd}, J=8.0,4.0 \mathrm{~Hz}$, $1 \mathrm{H}$, quinoline-H), $7.93 \sim 7.96(\mathrm{~m}, 1 \mathrm{H}, \mathrm{ArH}), 8.02(\mathrm{~d}, J=$ $8.0 \mathrm{~Hz}, 1 \mathrm{H}$, quinoline-H), $8.13(\mathrm{~d}, J=4.0 \mathrm{~Hz}, 2 \mathrm{H}$, quinoline-H and $\mathrm{CONH}), 8.22(\mathrm{~d}, J=4.0 \mathrm{~Hz}, 1 \mathrm{H}$, quinoline-H), 8.37 (s, 1H, ArH); HRMS calcd for $\mathrm{C}_{23} \mathrm{H}_{12} \mathrm{ClF}_{2} \mathrm{~N}_{3} \mathrm{O} 420.0710$, found 420.0746 . 
6-氯-2-(2,6-二氟苯基)- $N$-(3-氧基苯基)喹啉-4-甲酰 胺(N9): 白色晶体, 收率 79\%. m.p. 276 279 ${ }^{\circ} \mathrm{C} ;{ }^{1} \mathrm{H}$ NMR (DMSO- $\left.d_{6}, 400 \mathrm{MHz}\right) \delta: 7.35(\mathrm{t}, J=12.0 \mathrm{~Hz}, 2 \mathrm{H}$, ArH), $7.62 \sim 7.67(\mathrm{~m}, 3 \mathrm{H}, \mathrm{ArH}), 7.96(\mathrm{dd}, J=8.0,1.6 \mathrm{~Hz}$, $1 \mathrm{H}$, quinoline-H), $7.99 \sim 8.01(\mathrm{~m}, 1 \mathrm{H}, \mathrm{ArH}), 8.07(\mathrm{~s}, 1 \mathrm{H}$, quinoline-H), $8.22(\mathrm{~d}, J=8.0 \mathrm{~Hz}, 1 \mathrm{H}$, quinoline- $\mathrm{H}), 8.29$ (t, $J=2.0 \mathrm{~Hz}, 1 \mathrm{H}, A r H), 8.30(\mathrm{~d}, J=1.6 \mathrm{~Hz}, 1 \mathrm{H}$, quinoline-H), $11.20(\mathrm{~s}, 1 \mathrm{H}, \mathrm{CONH})$; HRMS calcd for $\mathrm{C}_{23} \mathrm{H}_{12} \mathrm{ClF}_{2} \mathrm{~N}_{3} \mathrm{O} 420.0710$, found 420.0745 .

6-氯-2-(2,6-二氟苯基)- $N$-芐基喹啉-4-甲酰胺 (N10): 白色晶体, 收率 $60 \%$. m.p. $177 \sim 181{ }^{\circ} \mathrm{C}$; ${ }^{1} \mathrm{H}$ NMR $\left(\mathrm{CDCl}_{3}, 400 \mathrm{MHz}\right) \delta: 4.74\left(\mathrm{~d}, J=4.0 \mathrm{~Hz}, 2 \mathrm{H}, \mathrm{CH}_{2}\right), 6.48$ (t, $J=8.0 \mathrm{~Hz}, 1 \mathrm{H}, \mathrm{ArH}), 7.04$ (t, $J=8.0 \mathrm{~Hz}, 2 \mathrm{H}, \mathrm{ArH}$ ), $7.32 \sim 7.40(\mathrm{~m}, 6 \mathrm{H}$, ArH and $\mathrm{CONH}), 7.63(\mathrm{~s}, 1 \mathrm{H}$, quinoline-H), $7.73(\mathrm{dd}, J=8.0,1.6 \mathrm{~Hz}, 1 \mathrm{H}$, quinoline-H), $8.12(\mathrm{~d}, J=8.0 \mathrm{~Hz}, 1 \mathrm{H}$, quinoline-H), $8.36(\mathrm{~d}, J=1.6 \mathrm{~Hz}$, $1 \mathrm{H}$, quinoline- $\mathrm{H}$ ); HRMS calcd for $\mathrm{C}_{23} \mathrm{H}_{15} \mathrm{ClF}_{2} \mathrm{~N}_{2} \mathrm{O}$ 409.0914, found 409.0958.

6-氯-2-(2,6-二氟苯基)- $N$-(2-氟苯基)喹啉-4-甲酰胺 (N11): 淡黄色晶体, 收率 58\%. m.p. 193 198 ${ }^{\circ} \mathrm{C} ;{ }^{1} \mathrm{H}$ NMR $\left(\mathrm{CDCl}_{3}, 400 \mathrm{MHz}\right) \delta: 7.07$ (t, $\left.J=8.0 \mathrm{~Hz}, 2 \mathrm{H}, \mathrm{ArH}\right)$, $7.15 \sim 7.25$ (m, 3H, ArH), $7.42 \sim 7.47$ (m, 1H, ArH), 7.77 (dd, $J=8.0,1.2 \mathrm{~Hz}, 1 \mathrm{H}$, quinoline-H), $7.80(\mathrm{~s}, 1 \mathrm{H}$, quinoline-H), $8.04(\mathrm{~s}, 1 \mathrm{H}, \mathrm{CONH}), 8.18(\mathrm{~d}, J=8.0 \mathrm{~Hz}, 1 \mathrm{H}$, quinoline-H), $8.40(\mathrm{~d}, J=1.2 \mathrm{~Hz}, 1 \mathrm{H}$, quinoline-H), 8.50 ( $\mathrm{t}, J=8.0 \mathrm{~Hz}, 1 \mathrm{H}, \mathrm{ArH}$ ); HRMS calcd for $\mathrm{C}_{22} \mathrm{H}_{12} \mathrm{ClF}_{3} \mathrm{~N}_{2} \mathrm{O}$ 413.0663, found 413.0710.

6-氯-2-(2,6-二氟苯基)- $N$-(4-氟苯基)喹啉-4-甲酰胺 (N12): 黄色晶体, 收率 53\%. m.p. $177 \sim 183{ }^{\circ} \mathrm{C} ;{ }^{1} \mathrm{H}$ NMR $\left(\mathrm{CDCl}_{3}, 400 \mathrm{MHz}\right) \delta: 7.02$ (t, $\left.J=8.0 \mathrm{~Hz}, 2 \mathrm{H}, \mathrm{ArH}\right)$, $7.07 \sim 7.10(\mathrm{~m}, 2 \mathrm{H}, \operatorname{ArH}), 7.38 \sim 7.43(\mathrm{~m}, 1 \mathrm{H}, \operatorname{ArH})$, $7.64 \sim 7.67$ (m, 2H, ArH), 7.69 (s, 1H, quinoline-H), 7.73 (dd, $J=8.0,1.6 \mathrm{~Hz}, 1 \mathrm{H}$, quinoline-H), $8.12(\mathrm{~d}, J=4.0 \mathrm{~Hz}$, $1 \mathrm{H}$, quinoline-H), $8.22(\mathrm{~s}, 1 \mathrm{H}, \mathrm{CONH}), 8.29$ (d, $J=1.6 \mathrm{~Hz}$, $1 \mathrm{H}$, quinoline- $\mathrm{H}$ ); HRMS calcd for $\mathrm{C}_{22} \mathrm{H}_{12} \mathrm{ClF}_{3} \mathrm{~N}_{2} \mathrm{O}$ 413.0663, found 413.0705.

6-氯-2-(2,6-二氟苯基)- $N$-(2-氯苯基)喹啉-4-甲酰胺 (N13): 白色晶体, 收率 61\%. m.p. 207 212 ${ }^{\circ} \mathrm{C} ;{ }^{1} \mathrm{H}$ NMR $\left(\mathrm{CDCl}_{3}, 400 \mathrm{MHz}\right) \delta: 7.10(\mathrm{t}, J=8.0 \mathrm{~Hz}, 2 \mathrm{H}, \mathrm{ArH})$, $7.18(\mathrm{td}, J=12.0 \mathrm{~Hz}, 1 \mathrm{H}, \mathrm{ArH}), 7.40 \sim 7.47$ (m, 3H, ArH), 7.78 (dd, $J=8.0,1.6 \mathrm{~Hz}, 1 \mathrm{H}$, quinoline-H), 7.85 (s, $1 \mathrm{H}$, quinoline-H), $8.20(\mathrm{~d}, J=8.0 \mathrm{~Hz}, 1 \mathrm{H}$, quinoline-H), 8.30 (d, $J=1.2 \mathrm{~Hz}, 1 \mathrm{H}, \mathrm{CONH}), 8.46(\mathrm{~d}, J=1.6 \mathrm{~Hz}, 1 \mathrm{H}$,
quinoline-H), $8.60(\mathrm{~d}, J=4.0 \mathrm{~Hz}, 3 \mathrm{H}, \mathrm{ArH})$; HRMS calcd for $\mathrm{C}_{22} \mathrm{H}_{12} \mathrm{Cl}_{2} \mathrm{~F}_{2} \mathrm{~N}_{2} \mathrm{O} 429.0368$, found 429.0410 .

辅助材料(Supporting Information) 提供了核磁共振 氢谱 $\left({ }^{1} \mathrm{H}\right.$ NMR) 和高分辨质谱(HRMS). 这些材料可以免 费从本刊网站(http://sioc-journal.cn/)上下载.

\section{References}

[1] Liu, X. H.; Xu, X. Y.; Tan, C. X.; Weng, J. Q.; Xin, J. H.; Chen, J. Pest Manage Sci. 2015, 71, 292.

[2] Liu, X. H.; Sun, Z. H.; Yang, M. Y.; Tan, C. X.; Weng, J. Q.; Zhang, Y. G.; Ma, Y. Chem. Biol. Drug Des. 2014, 84, 342.

[3] Nicholson, A. N.; Stone, B. M.; Clarke C. H. J. Clin. Pharmacol. 1977, 4, 567.

[4] Zayed, M. E. M.; El-Shishtawy, R. M.; Elroby, S. A.; Obaid, A. Y.; Al-amshany, Z. M. Int. J. Mol. Sci. 2015, 16, 3804.

[5] Wang, L.; Świtalska, M.; Wang, N.; Du, Z.-J.; Fukumoto, Y.; Diep, N. K.; Kiguchi, R.; Nokami, J.; Wietrzyk, J.; Inokuchi, T. Molecules 2014, 19, 19021.

[6] Fragoulis, G.; Merli, A.; Reeves, G.; Meregalli, G.; Stenberg, K.; Tanaka, T.; Capri, E. Pest Manage. Sci. 2011, 67, 656

[7] Liu, C. L. World Pesticide: Fungicide, Chemical Industry Press, Beijing, 2006, p. 251 (in Chinese).

(刘长令, 世界农药大全杀菌剂卷, 化学工业出版社, 北京, 2006, p. 251.)

[8] Nakano, H.; Masumizu, T. JP 2009091320, 2009 [Chem. Abstr. 2010, 150, 472698].

[9] Hackler, R. E.; Jourdan G. P.; Johnson, P. L.; Thoreen, B. R.; Samaritoni, J. G. WO 9304580, 1993 [Chem. Abstr. 1993, 119, 139111].

[10] (a) Liu, X. H.; Weng, J. Q.; Wang, B. L.; Li, Y. H.; Tan, C. X.; Li, Z. M. Chem. Res. Intermed. 2014, 40, 2605.

(b) Yang, M. Y.; Zhao, W.; Sun, Z. H.; Tan, C. X.; Weng, J. Q.; Liu, X. H. Lett. Drug Des. Discovery 2015, 12, 314.

(c) Xie, F.; Liu, T. T.; Yang, G.; Yuan, J.; Kong, X. L.; Xu, T. M.; Tan, C. X. Chin. J. Org. Chem. 2013, 33, 2596 (in Chinese). (谢峰, 刘婷婷, 杨果, 袁静, 孔小林, 许天明, 谭成侠, 有机化 学, 2013, 33, 2596.)

(d) Yan, S. L.; Yang, M. Y.; Sun, Z. H.; Min, L. J.; Tan, C. X.; Weng, J. Q.; Wu, H. K.; Liu, X. H. Lett. Drug Des. Discov. 2014, 11,940 .

(e) Sun, G.-X.; Sun, Z.-H.; Yang, M.-Y.; Liu, X.-H.; Ma, Y.; Wei, Y.-Y. Molecules 2013, 18, 14876.

[11] Karen, L.; Daniel, D. S. Synthesis 1993, 993.

[12] Yu, M. Q.; Liu, G. Y.; Y, X. R.; Chen, Q. Y.; Di, N. Fine Chem. Intermed. 2011, 41, 1 (in Chinese).

(喻名强, 刘广义, 伊兴荣, 陈秦豫, 狄宁, 精细化工中间体, 2011, 41, 1.)

[13] (a) Liu, X. H.; Pan, L.; Tan, C. X.; Weng, J. Q.; Wang, B. L.; Li, Z. M. Pest. Biochem. Physiol. 2011, 101, 143.

(b) Wu, R.; Zhu, C.; Du, X. J.; Xiong, L. X.; Yu, S. J.; Liu, X. H.; Li, Z. M.; Zhao, W. G. Chem. Cent. J. 2012, 6, 99.

[14] Liu, X. H.; Tan, C. X.; Weng, J. Q. Phosphorus, Sulfur, Silicon Relat. Elem. 2011, 186, 558. 